\title{
On the approximation of fixed points for a new class of generalized Berinde mappings
}

\section{BESSEM SAMET}

\section{ABSTRACT.}

In this paper, we introduce a new class of operators, for which a fixed point theorem is proven. This class of mappings is very large and unifies several classes of contractive type operators from the literature, including Berinde mappings. Such fact is proven via a comparison with various metrical contractive type mappings.

Acknowledgements. The author would like to extend his sincere appreciation to the Deanship of Scientific Research at King Saud University for its funding of this research through the International Research Group Project no. IRG14-04.

\section{REFERENCES}

[1] Berinde, V., Approximating fixed points of weak $\varphi$-contractions using the Picard iteration, Fixed Point Theory., 4 (2003), No. 2, 131-142

[2] Berinde, V., Approximating fixed points of weak contractions using the Picard iteration, Nonlinear Anal. Forum., 9 (2004), 43-54

[3] Berinde, V., Iterative approximation of fixed points, Vol. 1912, Berlin, Germany, Springer, 2007

[4] Berinde, V., Stability of Picard iteration for contractive mappings satisfying an implicit relation, Carpathian J. Math., 27 (2011), No. 1, 13-23

[5] Berinde, V. and Pacurar, M., Iterative approximation of fixed points of single-valued almost contractions, in Fixed Point Theory and Graph Theory: Foundations and Integrative Approaches by Monther Alfuraidan (Editor), Qamrul Ansari (Editor), Academic Press, 2016

[6] Chatterjea, S. K., Fixed-point theorems, C. R. Acad. Bulgare Sci., 25 (1972), 727-730

[7] Ćirić, Lj. B., A generalization of Banach's contraction principle, Proc. Am. Math. Soc., 45 (1974), 267-273

[8] Ćirić, Lj. B., On some maps with a nonunique fixed point, Pub. Inst. Math., 17 (1974), 52-58

[9] Dass, B. K. and Gupta, S., An extension of Banach contraction principle through rational expressions, Inidan J. Pure Appl. Math., 6 (1975), No. 12, 1455-1458

[10] Kannan, R., Some results on fixed points, Bull. Calcutta Math. Soc., 10 (1968), 71-76

[11] Popa, V., Fixed point theorems for implicit contractive mappings, Stud. Cerc. St. Ser. Mat. Univ. Bacau., 7 (1997), $127-133$

[12] Rus, I. A., Picard operator and applications, Babes-Bolyai Univ., 1996

[13] Samet, B., Vetro, C. and Vetro, P., Fixed point theorems for $\alpha$ - $\psi$-contractive type mappings, Nonlinear Anal., 75 (2012), No. 4, 2154-2165

[14] Zamfirescu, T., Fix point theorems in metric spaces, Arch. Math. (Basel), 23 (1972), 292-298

DePARTMENT OF MATHEMATics

COLLEge of SCIENCE

KING SAUD UNIVERSITY

P. O. BOX 2455, RIYADH 11451, SAUdi ARABIA

E-mail address: bsamet@ksu.edu.sa

Received: 27.02.2016; In revised form: 29.06.2016; Accepted: 30.06.2016

2010 Mathematics Subject Classification. 47H10.

Key words and phrases. Metric space, fixed point, $\alpha$-continuous, $\alpha$-Cauchy, $\alpha$-complete, Picard iteration, convergence. 